\title{
New insights into the morphology, reproduction and distribution of the large-tuberculate octopus Graneledone macrotyla from the Patagonian slope
}

\author{
ÁNGEL GUERRA ${ }^{1}$, ÁLVARO ROURA ${ }^{1}$, MARIA PILAR SIEIRO ${ }^{1}$, JULIO M. PORTELA ${ }^{2}$ \\ and JOSÉ LUIS DEL RÍO ${ }^{2}$ \\ ${ }^{1}$ Instituto de Investigaciones Marinas, CSIC, Eduardo Cabello 6, 36208 Vigo, Spain. E-mail: angelguerra@iim.csic.es \\ ${ }^{2}$ Instituto Español de Oceanografía (IEO), C. O. de Vigo, P.O. Box 1552, 36200 Vigo, Spain.
}

\begin{abstract}
SUMMARY: The new information reported in this paper is based on 11 specimens of the large-tuberculate octopus Graneledone macrotyla. These specimens were caught in bottom trawl surveys ATLANTIS 2009 and 2010 carried out on the Patagonian slope off the Argentinean Economic Exclusive Zone between 24 February and 1 April 2009 and from 9 March to 5 April 2010 respectively. A new diagnosis and a complete description of the species are provided. This is the first time that stylets, beaks and spermatophores are described. This is also the first time in which mature females have been studied and the female genitalia described. Like other eledonid octopods, G. macrotyla does not have spermathecae in the oviducal glands. The presence of fertilized eggs inside the ovary suggests that fertilization takes place within the ovary. The simultaneous occurrence of oocyte cohorts at different oogenic stages suggests that the species is a multiple spawner. $G$. macrotyla inhabits shallower waters on the Patagonian slope (475-921 m) than in the subantartic area (1647-2044 m). From a biogeographical point of view, our data show that G. macrotyla inhabits the plume of cold subantarctic waters, which is pushed far north into the southwestern Atlantic by the Falkland (Malvinas) Current.
\end{abstract}

Keywords: Graneledone macrotyla, Cephalopoda, systematic, reproduction, biogeography, Patagonian slope, southwest Atlantic.

RESUMEN: NUEVA INFORMACIÓN SOBRE LA MORFOLOGÍA, REPRODUCCIÓN Y DISTRIBUCIÓN DEL PULPO MEGATUBERCULADO GRANELEDONE MACROTYLA DEL TALUD CONTINENTAL PATAGÓNICO. - La novedosa información que se proporciona en este trabajo se basa en 11 ejemplares del pulpo megatuberculado Graneledone macrotyla. Esos ejemplares se capturaron en las campañas de prospección ATLANTIS 2009 y 2010, realizadas con arte de arrastre bentónico en el talud patagónico por fuera de la Zona Económica Exclusiva de Argentina, entre el 24 de febrero y el 1 de abril de 2009 y desde el 9 de marzo hasta el 5 de abril de 2010, respectivamente. Se ofrece una nueva diagnosis y una descripción completa de la especie. Esta es la primera vez en que se describen los estiletes, picos y espermatóforos. También es totalmente novedoso el estudio de hembras maduras, lo que ha permitido la descripción de su órgano genital. Como ocurre en otros pulpos de la subfamilia Eledoninae, G. macrotyla carece de espermatecas en las glándulas oviductales. La presencia de huevos fecundados en el ovario sugiere que la fecundación acontece dentro de este órgano. La presencia simultánea de varias cohortes de ovocitos de diferente tamaño sugiere que se trata de una especie cuya freza es múltiple. G. macrotyla vive en aguas más someras del talud patagónico $(475-921 \mathrm{~m})$ que en la región subantártica (1647-2044 m). Estos datos muestran que G. macrotyla vive en la pluma de aguas subantárticas, que transportan agua fría hacia el norte del Atlántico sudoccidental a través de la corriente de Malvinas (Falkland).

Palabras clave: Graneledone macrotyla, Cephalopoda, sistemática, reproducción, biogeografía, talud Patagónico, Atlántico sudoeste.

\section{INTRODUCTION}

The large-tuberculate octopus Graneledone macrotyla Voss, 1976 was first described on the basis of a single specimen: a female, mantle length $34.5 \mathrm{~mm}$, caught by the USNS ELTANIN at Station 1592, $54^{\circ} 43^{\prime} \mathrm{S}, 55^{\circ} 30^{\prime} \mathrm{W}$ in $1647-2044 \mathrm{~m}$ depth with a 10-foot Blake trawl on 14 March 1966 (Voss 1976). 
TABLE 1. - Details of trawls conducted during the ATLANTIS 2009 and ATLANTIS 2010 surveys in the high seas of the southwest Atlantic in which Graneledone macrotyla specimens were caught. HN': Haul Number; SST: Sea Surface Temperature; SBT: Sea Bottom Temperature; - : data not recorded; $\mathrm{N}^{\circ} \mathrm{S}$ : number of specimens in haul.

\begin{tabular}{|c|c|c|c|c|c|c|c|c|c|}
\hline \multirow[t]{2}{*}{$\mathrm{HN}^{\circ}$} & \multirow[t]{2}{*}{ Date } & \multicolumn{2}{|c|}{ Latitude (S) } & \multicolumn{2}{|c|}{ Longitude (W) } & \multicolumn{2}{|c|}{ Depth (m) } & \multirow[t]{2}{*}{ SST/SBT } & \multirow[t]{2}{*}{$\mathrm{N}^{\circ} \mathrm{S}$} \\
\hline & & Initial & Final & Initial & Final & Initial & Final & & \\
\hline 74 & $15 / 03 / 2009$ & $47^{\circ} 46.87$ & $47^{\circ} 45.31^{\prime}$ & $59^{\circ} 39.71^{\prime}$ & $59^{\circ} 38.99^{\prime}$ & 774 & 788 & $10.3 / 3.1$ & 1 \\
\hline 108 & $21 / 03 / 2009$ & $45^{\circ} 19.41$ & $45^{\circ} 17.84^{\prime}$ & $59^{\circ} 41.11^{\prime}$ & $59^{\circ} 41.03^{\prime}$ & 870 & 870 & $9.9 / 3.0$ & 1 \\
\hline 47 & $21 / 03 / 2010$ & $46^{\circ} 56.58^{\prime}$ & $46^{\circ} 55.21^{\prime}$ & $60^{\circ} 28.75^{\prime}$ & $60^{\circ} 27.90^{\prime}$ & 475 & 480 & $8.5 /-$ & 1 \\
\hline 55 & $23 / 03 / 2010$ & $47^{\circ} 30.68^{\prime}$ & $47^{\circ} 29.13^{\prime}$ & $60^{\circ} 29.38^{\prime}$ & $60^{\circ} 29.60^{\prime}$ & 489 & 495 & $8.4 /-$ & 1 \\
\hline 68 & $26 / 03 / 2010$ & $47^{\circ} 24.39^{\prime}$ & $47^{\circ} 22.91^{\prime}$ & $59^{\circ} 43.49^{\prime}$ & $59^{\circ} 44.04^{\prime}$ & 837 & 838 & $7.5 /-$ & 3 \\
\hline 92 & 02/04/2010 & $45^{\circ} 16.98^{\prime}$ & $45^{\circ} 15.46^{\prime}$ & $59^{\circ} 37.70^{\prime}$ & $59^{\circ} 38.03^{\prime}$ & 918 & 921 & 7.8/- & 1 \\
\hline 94 & $02 / 04 / 2010$ & $45^{\circ} 08.02^{\prime}$ & $45^{\circ} 06.80^{\prime}$ & $59^{\circ} 49.98^{\prime}$ & $59^{\circ} 50.19^{\prime}$ & 779 & 772 & $8.2 /-$ & 3 \\
\hline
\end{tabular}

Furuya and Hochberg (2001) described a new species of parasite, Dicyemennea dorycephalum, from the holotype. Kubodera and Okutani (1994) provided a supplementary description and some remarks on this octopus species, including details of the male reproductive organs and new distribution records, based on two specimens caught south of $45^{\circ} \mathrm{S}$ along the southeastern Patagonian slope.

In spite of this additional information, the description of this species is still incomplete, mainly because the three specimens used were small (ML 34.5-67.0 $\mathrm{mm}$ ) and did not include any mature females.

The main aims of the present paper are to improve and complete the description of G. macrotyla and offer new insights into its geographic distribution and reproductive strategy.

\section{MATERIALS AND METHODS}

Eleven specimens of G. macrotyla were caught during two multidisciplinary research cruises conducted by the Instituto Español de Oceanografía (IEO) to assess the biomass of the main commercial stocks on the high seas of the southwest Atlantic. The bottom trawl surveys ATLANTIS 2009 and 2010 were carried out between 24 February and 1 April 2009 (Guerra et al. 2011) and from 9 March to 5 April 2010 respectively, on board the R/V Miguel Oliver (Table 1).

Catches were sorted immediately on board after capture. Cephalopods were stored in labelled plastic bags and frozen at $-20^{\circ} \mathrm{C}$. Specimens were subsequently transported from the R/V Miguel Oliver to the Instituto de Investigaciones Marinas (IIM, CSIC) in Vigo, Spain, for detailed examination. The material used in this work, as well as the entire collection of cephalopods collected in these two research cruises, are deposited in the Museo do Mar de Galicia (MDMG) at Vigo, Spain (www.museodomar.com/es).

After thawing at room temperature the specimens were identified and the sex was determined. Total length (TL), dorsal mantle length (ML) and wet weight were measured. The 11 specimens of G. macrotyla were then preserved in ethanol 70\%. All measurements were repeated on these preserved specimens. We used the morphometry defined by Roper and Voss (1983).

The histological study of the oviducal gland and oviducts was conducted on specimen MDMG
68B2011, whose gland had a maximum diameter of 27 $\mathrm{mm}$. These structures were fixed in a solution of $10 \%$ buffered formaldehyde at a formaldehyde/tissue ratio approximately equal to 50. After fixation the tissues were thoroughly rinsed with water. Each sample was then dehydrated in ethanol in increasing concentrations from 70 to $100 \%$, and embedded individually in a paraffin block $\left(58^{\circ} \mathrm{C}\right)$ according to standard histological techniques (Gabe 1968). Longitudinal and transverse sections were cut (6-8 $\mu \mathrm{m}$ thick) at different levels of each structure and stained with HARRY's hematoxylin-eosin. Sections were photographed and examined using an image analysis system (NIS-Elements 3.0).

The radula preparation procedure was as follows: tissues around the radular ribbon were digested with trypsine $1 \%$ during one day, and then the radula was mounted in glycerine, dehydrated in ethanol 96\%, cleared with xilol, stained with Orange $\mathrm{G}$ to $0.1 \%$ during 1 hour, and mounted in synthetic resin DPX. Due to teeth variations, especially in the rachidian tooth, the most common teeth pattern has been drawn. The mounted radula was photographed using a binocular microscope with a magnification of 60x coupled with an image analysis system (NIS-Elements 3.0). Radular nomenclature follows Nixon (1995).

Given the lack of genetic data for this species, tissue samples have been submitted to the International Barcode of Life (iBOL) in order to amplify the barcoding cytochrome oxidase subunit I (COI) gene.

\section{SYSTEMATICS}

\section{Graneledone macrotyla Voss 1976}

(Figs 1-10, Tables 2-3)

Diagnosis. Medium size species, mature males from $84 \mathrm{~mm}$ ML and mature females from $110 \mathrm{~mm}$ ML, with distinctive sculpture. Dorsal surface of mantle, head and basal part of the arms covered by distinct complex papillose warts; 10-12 wart clusters across the dorsal mantle. Body muscular. Arms typically 1.8-2.5 times ML. Two tongue-shaped and tuberculate supraocular cirri on each eye, the posterior larger (10$14 \% \mathrm{ML}$ ) than the anterior one.

Description. Medium-sized octopodid; ML up to at least $110 \mathrm{~mm}$ for females; TL to at least $366 \mathrm{~mm}$ 

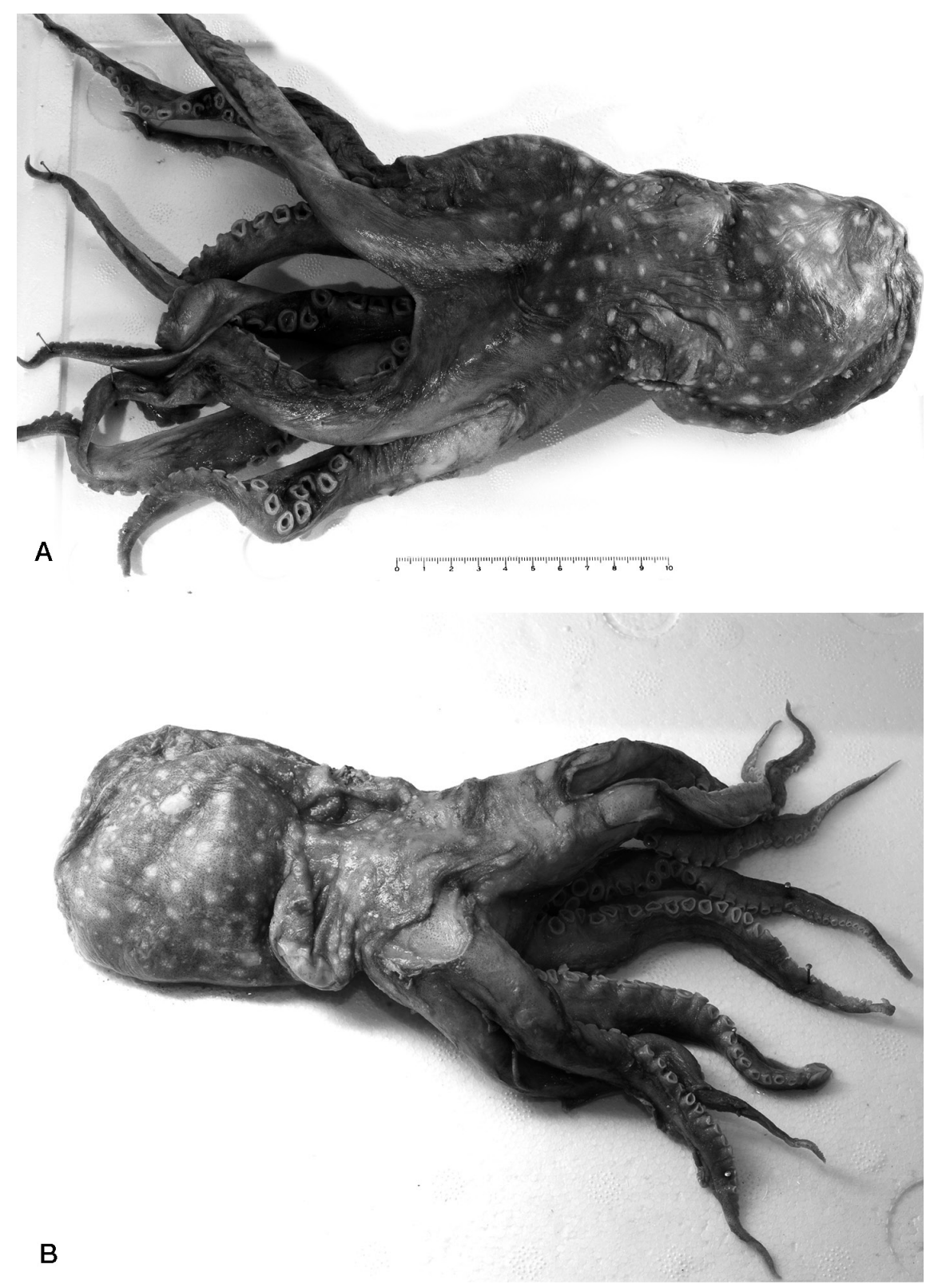

Fig. 1. - Graneledone macrotyla. A, dorsal view of the specimen 68A (female $98.2 \mathrm{~mm} \mathrm{ML}$ ); B, dorsal view of the male (specimen 74, male $84.8 \mathrm{~mm} \mathrm{ML})$;

for females. Mantle shape ovoid, somewhat dorsoventrally flattened and very wide (MWI 91.9-123.1) (Figs. $1 \mathrm{~A}$ and $1 \mathrm{~B})$. Mantle walls thick, muscular with some gelatinous material forming the outer layer. Stylets poorly developed (Fig. 2). Pallial aperture wide (occupying about $50 \%$ of the mantle circumference). Funnel broad-based and muscular (FuLI 50.0-69.3), stout and tubular; free portion variable in length; approximately 25-35\% funnel length (FFuLI 24.2-33.1). Funnel organ VV-shaped and stout with broad limbs, outer and median limbs of similar size but the outer limbs stouter than the median ones.

Head broad, slightly narrower than the mantle (HWI 73.3-92.3) except in the holotype (110.0). No constriction between head and body. Eyes large (EDI
34.0-51.6); large eye lens diameter (LDI 15.8-29.0). Two tongue-shaped and tuberculate supraocular cirri on each eye, posterior cirrus larger (10-14\% ML) than the anterior cirrus (5-6\% ML) (Fig. 3). Male and two females with posterior supraocular cirrus only, lengths of which varied from 9.9 to $12.0 \% \mathrm{ML}$. Slight constriction between mantle and arm bases.

Arms relatively short (ALI 150.9-271.2) and stout (AWI 19.7-22.7) gradually tapering to slender tips (Fig. 4A, B). Arms asymmetrical in length between right and left side. Most common arm formula on right side $2>1>3>4$. Suckers small (SDI 7.3-8.5), crowded together, arranged regularly in a single row (Fig. 4A, B). No enlarged suckers in either males or females. Number of suckers on each intact arm in submature 

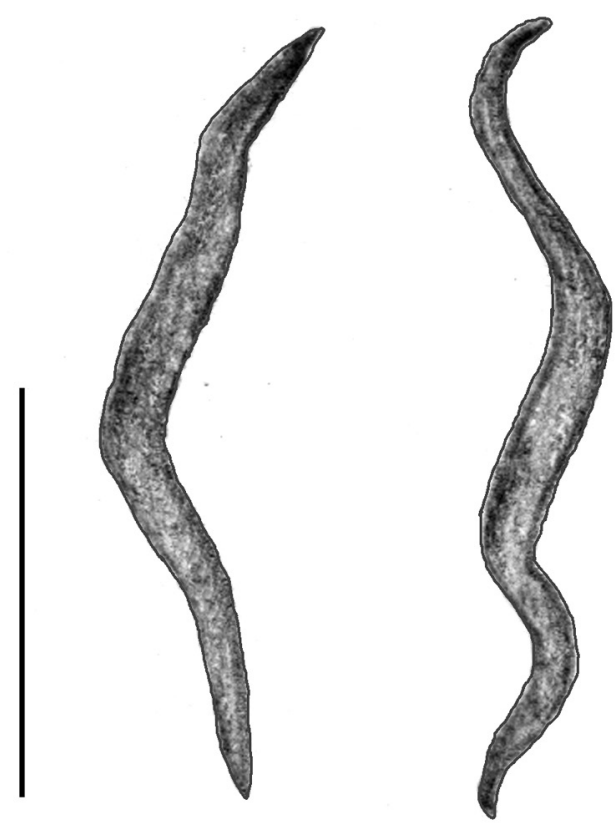

FIG. 2. - Stylets of Graneledone macrotyla. The asymmetry observed may be due to the body distortion caused by freezing. Bar: $1 \mathrm{~cm}$.

and mature animals comparatively low (SC from 32 to 53 in 21-37.5 ML animals and from 57 to 66 in 78.0110.0 ML animals).

Webs moderately deep (WDI 18.3-38.4), asymmetrical, formula variable, most commonly CBDAE on right side. Web extends as broad membrane up ventral side of each arm nearly to tip (Figs. 4A and 4B).

Third right arm of males hectocotylized, short (ALI3R 161.4), approximately $81 \%$ of length of opposite arm (Fig. 4B). Ligula small (LiLI 7.8-8.2), robust, not expanded with bluntly pointed tip (Fig. 5). Ligula groove deep, with 4-5 shallow slightly transverse ridges; lateral margins low. Calamus large (CaLI 49.1-67.1), raised well above ligula surface. 40 suckers on hectocotilized arm. Gills moderately large (GLI 20.3-30.4) with 7 lamellae, including terminal ones, on outer demibranch.

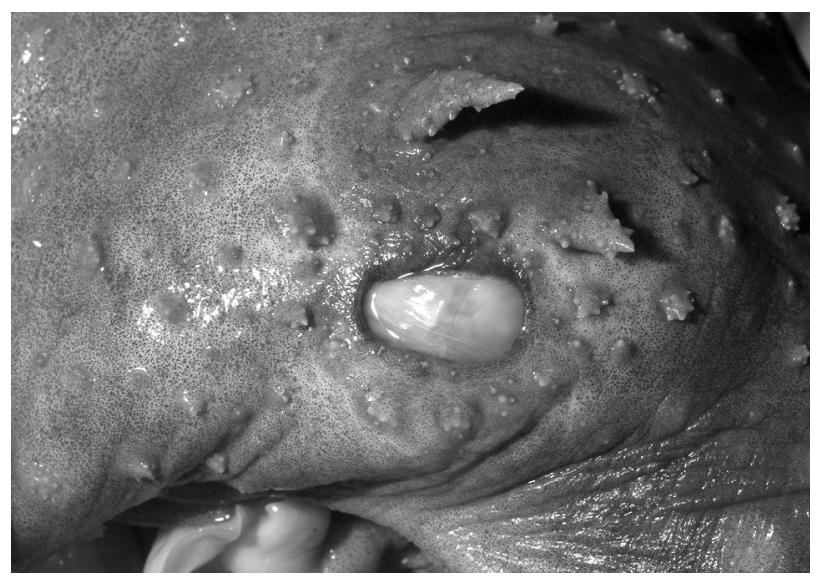

FIG. 3. - Graneledone macrotyla. Supraocular cirri (specimen 68A).

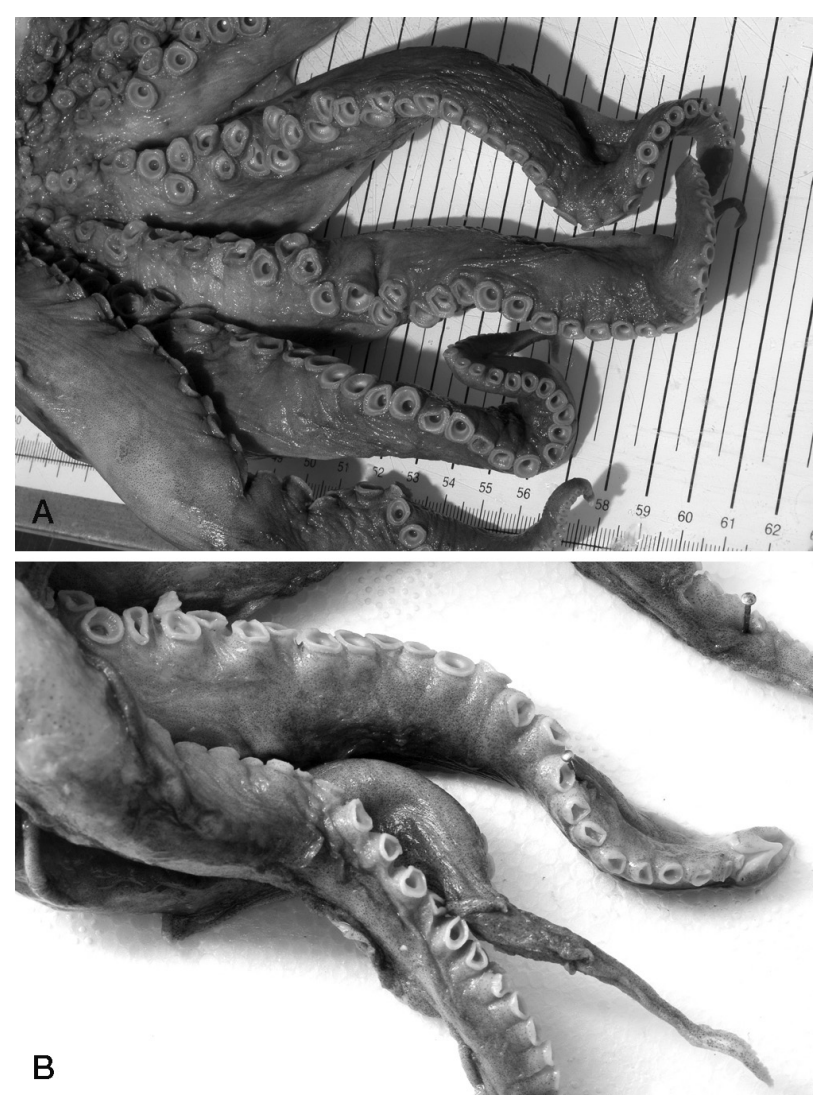

FIG. 4. - Graneledone macrotyla. A, arms, suckers and web of specimen $68 \mathrm{~A} ; \mathrm{B}$, hectocotylized arm of specimen 74 (III right).
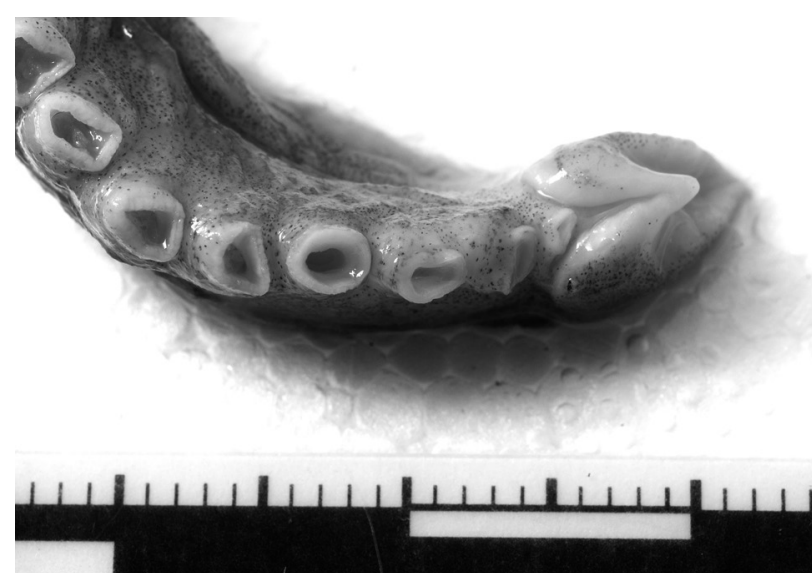

FIG. 5. - Graneledone macrotyla. Ligula and calamus of specimen 74.

Digestive tract illustrated in Figure 3d of Voss (1976). The buccal mass large. Anterior salivary glands small. Posterior salivary glands lanceolated and relatively small (16-17\% ML). Oesophagus stout and slightly dilated along much of its length, without diverticulum and apparently not forming a true crop. Dilated portion of oesophagus leads into a stronglydifferentiated two-part stomach and a distinct large and little spiral caecum. The intestine is large, slightly dilated, and bent almost double upon itself. The anal pore is round. Anal flaps absent. No trace of ink sac. 
TABLE 2. - Graneledone macrotyla. Measurements (in mm) of the female holotype (USNM727678) and the 11 specimens from the ATLANTIS 2009 and ATLANTIS 2010 research cruises. Spe, specimen number; MDMG, Museo do Mar de Galicia (Vigo, Spain); F, female; StM, stage of maturity; I, immature; Sm, submature; Mt, maturing; Ma, mature; S, sex; In, sex undifferentiated; M, male; F, female; ML, mantle length; TL, total length; BW, body weight (g); MW, mantle width; HW, head width; ED, eye diameter; LD, lens diameter; FuL, funnel length; FFuL, free funnel length; AL, arm length: 1,2,3 and 4; R: right; L: left; He: hectocotylized arm; AF, arm formula; LiL, ligula length; CaL, calamus length; ASC, arm sucker count, number of suckers on each arm; SD, sucker diameter; AW, arm width; W, web depth, A,B,C.D,E sectors on the right and left (L) side of the animal; WF, web formula; GL, gill length; GC, number of gill lamellae per demibranch; SpL, spermatophore length; D, damaged; Re, regenerating; --, not recorded.

\begin{tabular}{|c|c|c|c|c|c|c|c|c|c|c|c|c|}
\hline Spe & $\begin{array}{l}\text { USNM } \\
727678\end{array}$ & $\begin{array}{l}\text { MDMG } \\
742011\end{array}$ & $\begin{array}{l}\text { MDMG } \\
1082011\end{array}$ & $\begin{array}{l}\text { MDMG } \\
472011\end{array}$ & $\begin{array}{l}\text { MDMG } \\
552011\end{array}$ & $\begin{array}{l}\text { MDMG } \\
68 \mathrm{~A} 2011\end{array}$ & $\begin{array}{c}\text { MDMG } \\
68 \mathrm{~B} 2011\end{array}$ & $\begin{array}{l}\text { MDMG } \\
68 \mathrm{C} 2011\end{array}$ & $\begin{array}{l}\text { MDMG } \\
922011\end{array}$ & $\begin{array}{c}\text { MDMG } \\
94 \mathrm{~A} 2011\end{array}$ & $\begin{array}{c}\text { MDMG } \\
94 \mathrm{~B} 2011\end{array}$ & $\begin{array}{c}\text { MDMG } \\
94 C 2011\end{array}$ \\
\hline S/StM & $\mathrm{F}$ & MMa & In & FMt & FSm & FMt & FMa & FMt & In & In & In & In \\
\hline ML & 34.5 & 84.8 & 21.0 & 107.0 & 78.0 & 98.2 & 110.0 & 80.0 & 36.1 & 37.5 & 32.0 & 26.0 \\
\hline TL & 127.0 & 247.5 & 76.2 & 330.0 & 270.2 & 359.1 & 366 & 290.3 & 121.0 & 132 & 108.3 & 80.8 \\
\hline MW & 38.0 & 77.8 & 25.7 & 102.2 & 93.1 & 97.2 & 116.0 & 89.2 & 41.0 & 37.4 & 31.6 & 32.0 \\
\hline HW & 36.0 & 75.4 & 19.1 & 81.4 & 64.2 & 77.1 & 84.2 & 62.3 & 28.3 & 27.5 & 27.2 & 24.0 \\
\hline ED & -- & 28.1 & 10.2 & 48.2 & 27.2 & 43.2 & 48.8 & 40.6 & 18.3 & 19.2 & 16.5 & 13.3 \\
\hline LD & -- & 20.2 & 6.1 & 17.3 & 17.1 & 15.5 & 17.4 & 13.2 & 10.1 & 8.5 & 8.0 & 6.9 \\
\hline FuL & -- & 42.8 & 10.5 & 66.1 & 50.3 & 62.5 & 67.9 & 48.2 & 20.6 & 26.0 & 21.2 & 17.0 \\
\hline FFuL & -- & 22.4 & 6.8 & 26.9 & 21.7 & 24.4 & 29.5 & 26.5 & 10.9 & 10.4 & 8.0 & 6.3 \\
\hline AL1R & 81.0 & 159.8 & 37.7 & 208.6 & $112.8 \mathrm{R}$ & 244.0 & 248 & $\mathrm{Re}$ & 82.6 & 78.0 & 63.0 & 47.0 \\
\hline AL2R & 87.0 & 155.1 & 36.8 & 216.2 & 171.6 & 259.3 & 254 & $\mathrm{Re}$ & 83.3 & $\mathrm{Re}$ & 65.4 & $\mathrm{D}$ \\
\hline AL3R(He) & 83.0 & 136.9 & 33.2 & 215.3 & 169.3 & $\mathrm{Re}$ & 233 & 203.3 & 82.0 & $\mathrm{Re}$ & D & D \\
\hline AL4R & 80.0 & 146.5 & 31.7 & 198.1 & 160.6 & 266.0 & 229 & 182.0 & 75.4 & $\mathrm{Re}$ & 61.5 & $\mathrm{D}$ \\
\hline AL1L & -- & 152.3 & 39.4 & 189.4 & 164.0 & 265.0 & 245 & 211.2 & 94.7 & 85.0 & 61.0 & 55.4 \\
\hline AL2L & -- & 159.5 & 36.4 & 196.0 & 164.2 & 244.2 & 246 & 205.1 & $\mathrm{Re}$ & 86.0 & 64.0 & 61.9 \\
\hline AL3L & -- & 168.6 & $\operatorname{Re}$ & 217.6 & 160.1 & 265.2 & 232 & 192.4 & 80.3 & $\operatorname{Re}$ & 61.2 & 54.6 \\
\hline AL4L & -- & 158.2 & 37.4 & 198.3 & $149 \mathrm{R}$ & 266.3 & 264 & 181.3 & 80.6 & 87.0 & 65.4 & 50.3 \\
\hline LiL & -- & 6.6 & -- & -- & -- & -- & -- & -- & -- & -- & -- & -- \\
\hline $\mathrm{CaL}$ & -- & 4.3 & -- & -- & -- & -- & -- & -- & -- & -- & -- & -- \\
\hline AFR & 2.3.1.4 & $1.2 .4 .3(\mathrm{H})$ & -- & 2.3.1.4 & -- & 2.1 .3 .4 & 2.1 .3 .4 & -- & 2.1.3.4 & -- & -- & -- \\
\hline AFL & -- & 3.2.4.1 & -- & 3.4 .2 .1 & -- & 4.1 .3 .2 & 4.2 .1 .3 & 1.2 .3 .4 & -- & -- & 4.2 .3 .1 & 2.1.3.4 \\
\hline ASC A1R/L & -- & $62 / 60$ & $37 / 38$ & $63 / 60$ & $61 / 62$ & $63 / 65$ & $61 / 61$ & $\mathrm{Re} / 62$ & $40 / 41$ & $\mathrm{D} / 44$ & $41 / 44$ & $\mathrm{D} / 43$ \\
\hline A2 & -- & $63 / 61$ & $34 / 33$ & $63 / 65$ & $54 / 54$ & $65 / \mathrm{D}$ & $65 / 66$ & $\mathrm{Re} / 63$ & $40 / \mathrm{D}$ & $\mathrm{D} / 45$ & $45 / 52$ & $\mathrm{D} / 53$ \\
\hline $\mathrm{A} 3(\mathrm{H})$ & -- & $40 / 61$ & $34 / 32$ & $62 / 57$ & $61 / 62$ & $-/ 65$ & $65 / 63$ & $60 / 62$ & $45 / 45$ & $\mathrm{D} / \mathrm{D}$ & $45 / 47$ & $\mathrm{D} / 48$ \\
\hline A4 & -- & $60 / 58$ & $32 / 32$ & $60 / 59$ & $60 / 62$ & $57 / 64$ & $65 / 62$ & $65 / 64$ & $44 / 42$ & $\mathrm{D} / 47$ & $40 / 43$ & $\mathrm{D} / 46$ \\
\hline SD & 2.5 & 6.7 & 1.3 & 9.1 & 6.2 & 7.2 & 9.2 & 6.8 & 2.7 & 2.8 & 2.4 & 1.9 \\
\hline AW & 7.5 & 17.3 & -- & 22.5 & -- & 21.3 & 22.3 & 18.2 & 7.4 & 7.6 & 6.1 & 5.4 \\
\hline WD:A & 24.0 & 36.5 & 10.8 & 59.1 & 40.0 & 61.1 & 64.2 & 51.0 & 21.3 & 19.5 & 15.7 & 13.5 \\
\hline WD:BR/L & 24.0 & $45.9 / 46.4$ & $12.2 / 11.7$ & $63.3 / 75.2$ & $45.1 / 55.4$ & $78.2 / 62.1$ & $68.0 / 74.5$ & $72.2 / 52.8$ & $22.6 / 26.3$ & $22.4 / 22.4$ & $18.0 / 16.4$ & $17.4 / 15.4$ \\
\hline WD:CR/L & 28.0 & $45.7 / 36.6$ & $12.8 / 12.8$ & $72.6 / 83.5$ & $44.3 / 54.3$ & $97.0 / 100.1$ & $77.3 / 69.4$ & $71.2 / 74.5$ & $23.2 / 19.2$ & $\mathrm{D} / 22.3$ & $20.7 / 17.9$ & $19.2 / 19.0$ \\
\hline WD:DR/L & 25.5 & $47.1 / 42.7$ & $6.9 / 13.4$ & $79.1 / 57.3$ & $46.0 / 53.5$ & $76.2 / 99.1$ & $72.0 / 79.1$ & $69.8 / 72.6$ & $24.0 / 19.2$ & $\mathrm{D} / 19.2$ & $18.8 / 18.0$ & $14.4 / 15.5$ \\
\hline WD:E & 20.0 & 35.7 & 4.7 & 54.4 & 34.6 & 55.3 & 60.1 & 44.1 & 19.3 & 16.9 & 9.0 & 7.3 \\
\hline WFR & CDBAE & DBCAE & -- & DCBAE & -- & CBDAE & CDBAE & BCDAE & DCBAE & -- & CDBAE & CBDAE \\
\hline WFL & -- & BDCAE & -- & CBDAE & -- & CDBAE & DBCAE & CDBAE & BACDE & BCDAE & DCBAE & CDBAE \\
\hline GL & 8.0 & 25.8 & 6.3 & 23.1 & 22.5 & 23.4 & 24.8 & 16.2 & 10.4 & 9.0 & 9.4 & 7.5 \\
\hline GC & 7 & 7 & 7 & 7 & 7 & 7 & 7 & 7 & 7 & 7 & 7 & 7 \\
\hline $\mathrm{gL}$ & -- & -- & -- & 22.6 & -- & 20.5 & 25.5 & -- & -- & -- & -- & -- \\
\hline $\mathrm{EgW}$ & -- & -- & -- & 11.5 & -- & 9.6 & 11.7 & -- & -- & -- & -- & -- \\
\hline SpL & -- & 93.6 & -- & -- & -- & -- & -- & -- & -- & -- & -- & -- \\
\hline
\end{tabular}

Upper and lower mandibles or beaks well developed, not markedly different from other octopodids (Fig. 6). Radula shown in Figure 7

Well developed testis, first section of the duct system or proximal vas deferens narrow and convoluted, long spermatophoric gland, two strong coils proximally, the more distal one almost three times larger than the proximal one, long and thick second spermatophoric or accessory gland, thin Needham's or spermatophore storage sac, thin and poorly defined medial and distal spermatophoric ducts, terminal organ or penis curled, moderately long $(21.2 \%$ of the ML) and with a large diverticulum, sac-like and weakly coiled. Spermatophores very long (SpLI 110.4) and apparently produced in low numbers (2 in Needham's Sac in mature male MDMG742011, Table 2). Oral cap of the spermatophore hooked and very distinct (Fig. 8A and $8 \mathrm{~B})$.
Female genitalia illustrated in Fig. 9. Mature ovary oval-shaped. Proximal oviducts robust and short (6-9\% ML); distal oviducts relatively long (52-69\% ML) and

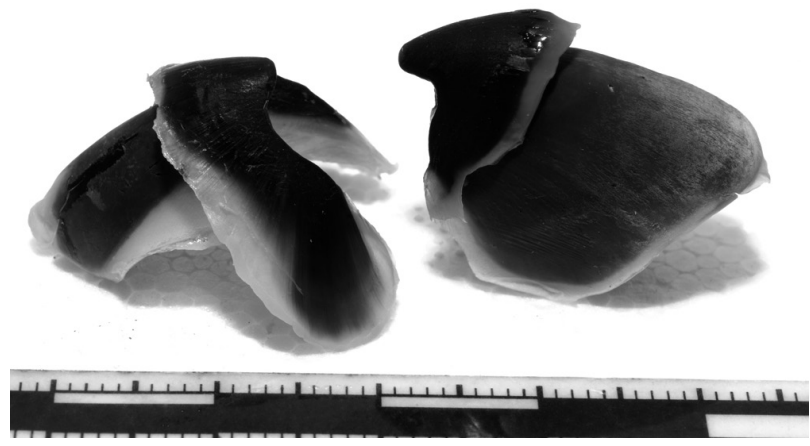

FIG. 6. - Graneledone macrotyla. Upper and lower beaks. 

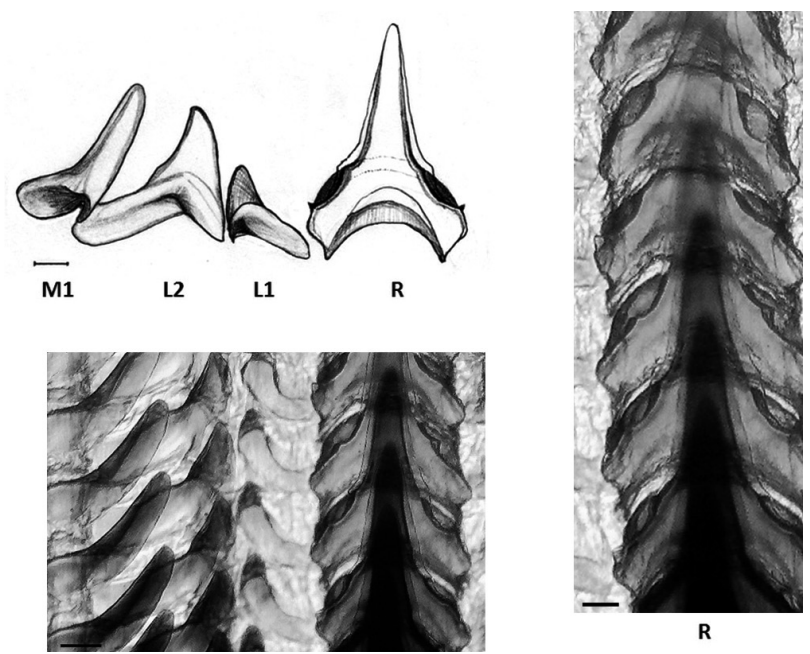

FIG. 7. - Radula of Graneledone macrotyla stained with Orange G and mounted in synthetic resin DPX. Drawing based on the most common teeth pattern. Photo taken with a binocular microscope with 60x magnification and image analysis system (NIS-Elements 3.0). Radular nomenclature follows Nixon (1995). R, radular tooth;

L1 and L2, lateral teeth; M1, first marginal tooth; Bars: $100 \mu \mathrm{m}$.

also robust (Fig 9A and 9B). Oviducal glands large; diameter varying from $15.4 \% \mathrm{ML}$ to $23.3 \% \mathrm{ML}$ in submature and mature animals respectively. Oviducts change colour with maturity from white-beige to uniformly dark grey-blueish when mature; no radiating chambers at any maturation stage.
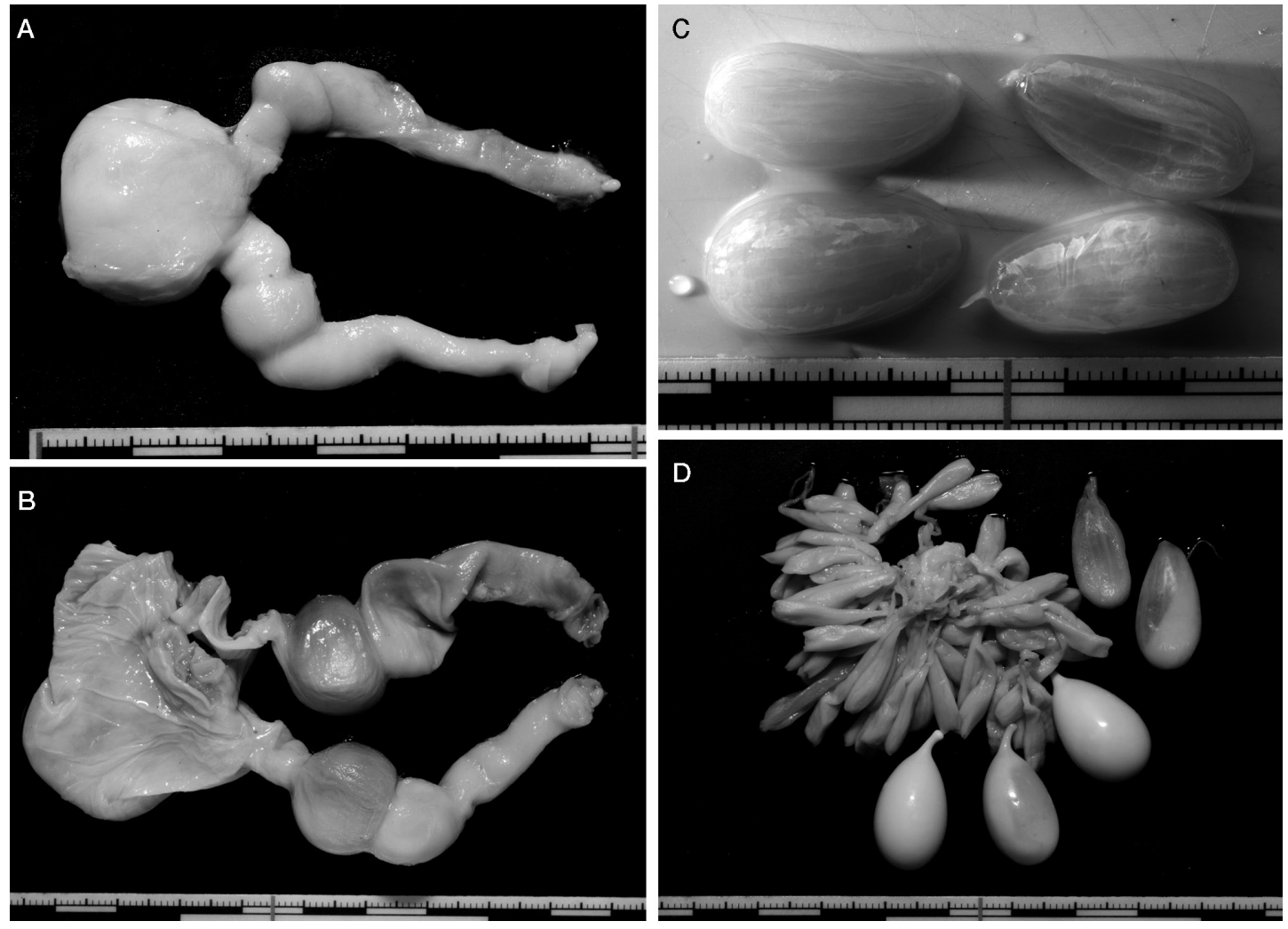

FIG. 9. - Graneledone macrotyla. A, female genitalia (specimen 55, $78 \mathrm{~mm} \mathrm{ML);} \mathrm{B,} \mathrm{female} \mathrm{genitalia} \mathrm{(specimen} \mathrm{47,} 107 \mathrm{~mm} \mathrm{ML);} \mathrm{C,} \mathrm{oocytes}$ (specimen 68B, $110 \mathrm{~mm} \mathrm{ML}$ ); D, oocytes and three ovarian eggs (specimen 47, $107 \mathrm{~mm} \mathrm{ML}$ ).

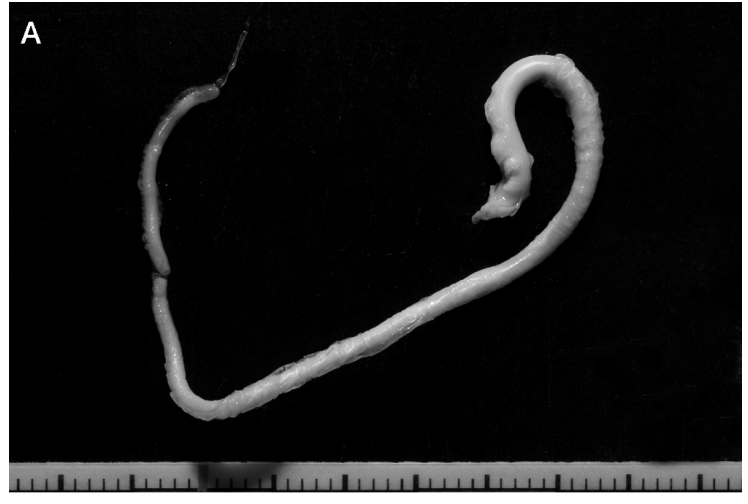

B

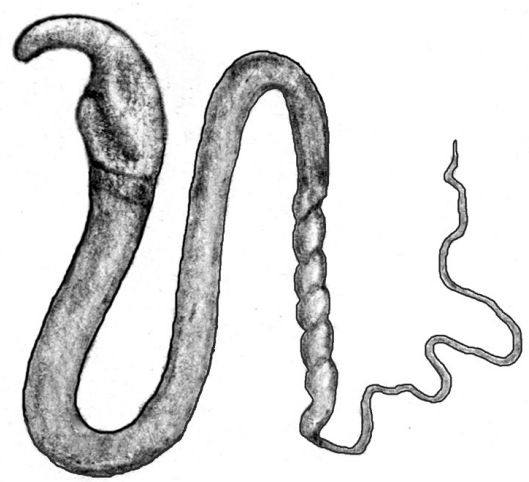

FIG. 8. - Graneledone macrotyla. Photo (A) and drawing (B) of the spermatophore. Bar: $1 \mathrm{~cm}$.

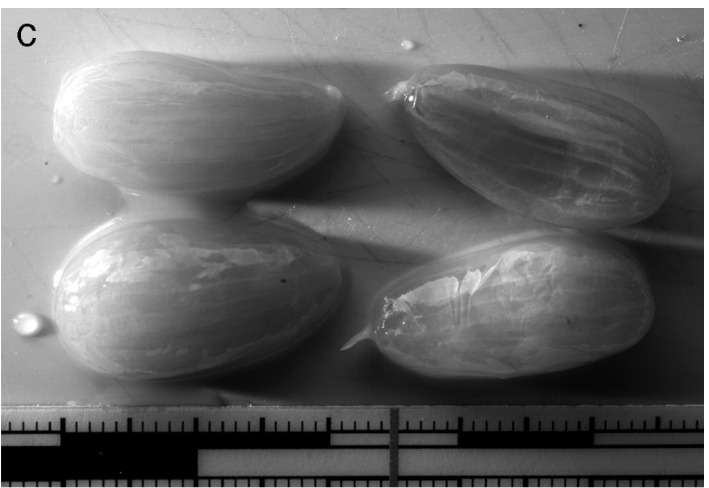


TABLE 3. - Graneledone macrotyla. Indices of the female holotype (USNM727678), the two specimens (67838 and 67839) in Kubodera and Okutani (1994) and the 11 specimens from the ATLANTIS 2009 and ATLANTIS 2010 research cruises. MDMG, Museo do Mar de Galicia (Vigo, Spain); MWI, mantle width index; HWI, head width index; EDI, eye diameter index; LDI, lens diameter index; FuLI, funnel length index; FFuLI, free funnel length index; ALI, arm length index; R, right; L, left; SDI, sucker diameter index; AWI, arm width index; WDI, web depth index in each sector (A-E); GLI, gill length index; EgLI, egg length index; EgWI, egg width index; LiLI, ligula length index; CaLI, calamus length index; $\mathrm{D}$, damaged; Re, regenerating; --, no calculated.

\begin{tabular}{|c|c|c|c|c|c|c|c|c|c|c|c|c|c|c|}
\hline Spe & $\begin{array}{l}\text { USNM } \\
727678\end{array}$ & 67838 & 67839 & $\begin{array}{l}\text { MDMG } \\
742011\end{array}$ & $\begin{array}{l}\text { MDMG } \\
1082011\end{array}$ & $\begin{array}{l}\text { MDMG } \\
472011\end{array}$ & $\begin{array}{c}\text { MDMG } \\
552011\end{array}$ & $\begin{array}{c}\text { MDMG } \\
\text { 68A2011 }\end{array}$ & $\begin{array}{c}\text { MDMG } \\
\text { 68B2011 }\end{array}$ & $\begin{array}{l}\text { MDMG } \\
68 \mathrm{C} 2011\end{array}$ & $\begin{array}{l}\text { MDMG } \\
922011\end{array}$ & $\begin{array}{c}\text { MDMG } \\
94 \mathrm{~A} 2011\end{array}$ & $\begin{array}{c}\text { MDMG } \\
94 \mathrm{~B} 2011\end{array}$ & $\begin{array}{l}\text { MDMG } \\
94 \mathrm{C} 2011\end{array}$ \\
\hline MWI & 110.0 & 87.3 & 115.4 & 91.9 & 122.3 & 95.5 & 119.4 & 99.0 & 105.4 & 111.5 & 113.6 & 99.7 & 98.7 & 123.1 \\
\hline HWI & 104.0 & 68.4 & 84.6 & 88.9 & 90.9 & 76.1 & 83.3 & 78.5 & 76.5 & 77.9 & 78.4 & 73.3 & 85.0 & 92.3 \\
\hline EDI & -- & -- & -- & 34.0 & 48.5 & 45.0 & 34.8 & 44.0 & 44.3 & 50.7 & 50.7 & 51.2 & 51.6 & 51.2 \\
\hline LDI & -- & -- & -- & 23.8 & 29.0 & 16.2 & 21.9 & 15.8 & 15.8 & 16.5 & 27.9 & 22.7 & 25.0 & 26.5 \\
\hline FuLI & -- & 39.6 & 49.2 & 50.5 & 50.0 & 61.8 & 64.8 & 63.6 & 61.7 & 60.3 & 54.9 & 69.3 & 66.3 & 65.4 \\
\hline FFuLI & -- & 20.6 & 23.8 & 26.4 & 32.4 & 25.2 & 27.8 & 24.8 & 26.8 & 33.1 & 30.2 & 27.7 & 25.0 & 24.2 \\
\hline ALI1 R & 234.8 & 201.5 & 261.5 & 188.4 & 179.5 & 194.9 & $\mathrm{Re}$ & 248.4 & 225.5 & $\mathrm{Re}$ & 228.8 & 208.0 & 196.9 & 180.8 \\
\hline ALI2 R & 252.2 & 198.5 & 238.5 & 182.9 & 175.2 & 202.1 & 220.0 & 264.1 & 230.9 & $\mathrm{Re}$ & 230.7 & $\mathrm{Re}$ & 204.4 & D \\
\hline ALI3 R & 240.6 & 149.3 & 238.5 & 161.4 & 158.1 & 201.2 & 217.1 & $\mathrm{Re}$ & 211.8 & 254.1 & 227.1 & $\mathrm{Re}$ & D & D \\
\hline ALI4 R & 231.9 & 179.1 & 223.1 & 172.8 & 150.9 & 185.1 & 205.9 & 270.9 & 208.2 & 227.5 & 208.9 & $\mathrm{Re}$ & 192.2 & D \\
\hline ALI1L & -- & 194.0 & 215.0 & 179.6 & 187.6 & 177.0 & 210.3 & 269.8 & 222.7 & 264.0 & 262.3 & 226.7 & 190.6 & 213.1 \\
\hline ALI2L & -- & 186.6 & 246.2 & 188.1 & 173.3 & 183.2 & 210.5 & 248.5 & 223.6 & 256.4 & $\mathrm{Re}$ & 229.3 & 200.0 & 238.1 \\
\hline ALI3L & -- & D & 238.5 & 198.8 & $\mathrm{R}$ & 203.4 & 205.3 & 270.1 & 210.9 & 240.5 & 222.4 & $\mathrm{Re}$ & 191.3 & 210.0 \\
\hline ALI4L & -- & 171.6 & 223.1 & 186.6 & 178.1 & 185.3 & $\mathrm{R}$ & 271.2 & 240.0 & 226.6 & 223.3 & 232.0 & 204.4 & 193.5 \\
\hline SDI & 7.2 & 7.2 & 9.1 & 7.9 & -- & 8.5 & 7.9 & 7.3 & 8.4 & 8.5 & 7.5 & 7.5 & 7.5 & 7.3 \\
\hline AWI & 21.7 & 17.9 & 24.6 & 20.4 & -- & 21.0 & -- & 21.7 & 20.3 & 22.7 & 20.5 & 20.3 & 19.7 & 20.8 \\
\hline WDIA & -- & -- & -- & 21.6 & 27.4 & 27.1 & 23.3 & 22.9 & 20.7 & 24.9 & 22.5 & 22.4 & 24.0 & 21.8 \\
\hline WDIB & -- & -- & -- & $28.7 / 27.5$ & $30.1 / 29.7$ & $29.3 / 34.5$ & $26.3 / 32.3$ & $29.4 / 23.1$ & $24.8 / 21.1$ & $35.5 / 25.7$ & $27.1 / 27.8$ & $25.7 / 25.7$ & $27.5 / 25.1$ & $28.1 / 24.9$ \\
\hline WDIC & 32.2 & 33.3 & 26.9 & $28.6 / 21.7$ & $32.5 / 32.5$ & $33.6 / 38.4$ & $25.8 / 31.6$ & $36.4 / 37.6$ & $33.4 / 37.3$ & $35.0 / 36.3$ & $27.9 / 20.3$ & $\mathrm{D} / 25.6$ & $31.6 / 27.4$ & $31.0 / 30.7$ \\
\hline WDID & -- & -- & -- & $29.5 / 25.3$ & $18.3 / 34.0$ & $36.6 / 26.3$ & $26.8 / 31.2$ & $28.6 / 37.2$ & $26.3 / 33.6$ & $34.3 / 35.4$ & $28.8 / 20.3$ & $\mathrm{D} / 22.1$ & $28.7 / 27.5$ & $23.3 / 25.0$ \\
\hline WDIE & -- & -- & -- & 21.2 & 11.9 & 25.0 & 20.2 & 20.8 & 19.1 & 21.5 & 20.4 & 19.4 & 13.8 & 11.8 \\
\hline GLI & -- & -- & -- & 30.4 & 30.0 & 21.6 & -- & 23.8 & 21.3 & 20.3 & 28.8 & 24.0 & 29.3 & 28.8 \\
\hline EgLI & -- & -- & -- & -- & -- & 21.1 & -- & 20.9 & 18.6 & -- & -- & -- & -- & -- \\
\hline EgWI & -- & -- & -- & -- & -- & 10.7 & -- & 9.8 & 8.7 & -- & -- & -- & -- & -- \\
\hline LiLI & -- & 8.2 & -- & 7.8 & -- & -- & -- & -- & -- & -- & -- & -- & -- & -- \\
\hline CaLI & -- & 49.1 & -- & 67.1 & -- & -- & -- & -- & -- & -- & -- & -- & -- & -- \\
\hline SpLI & -- & -- & -- & 110.4 & -- & -- & -- & -- & -- & -- & -- & -- & -- & -- \\
\hline
\end{tabular}
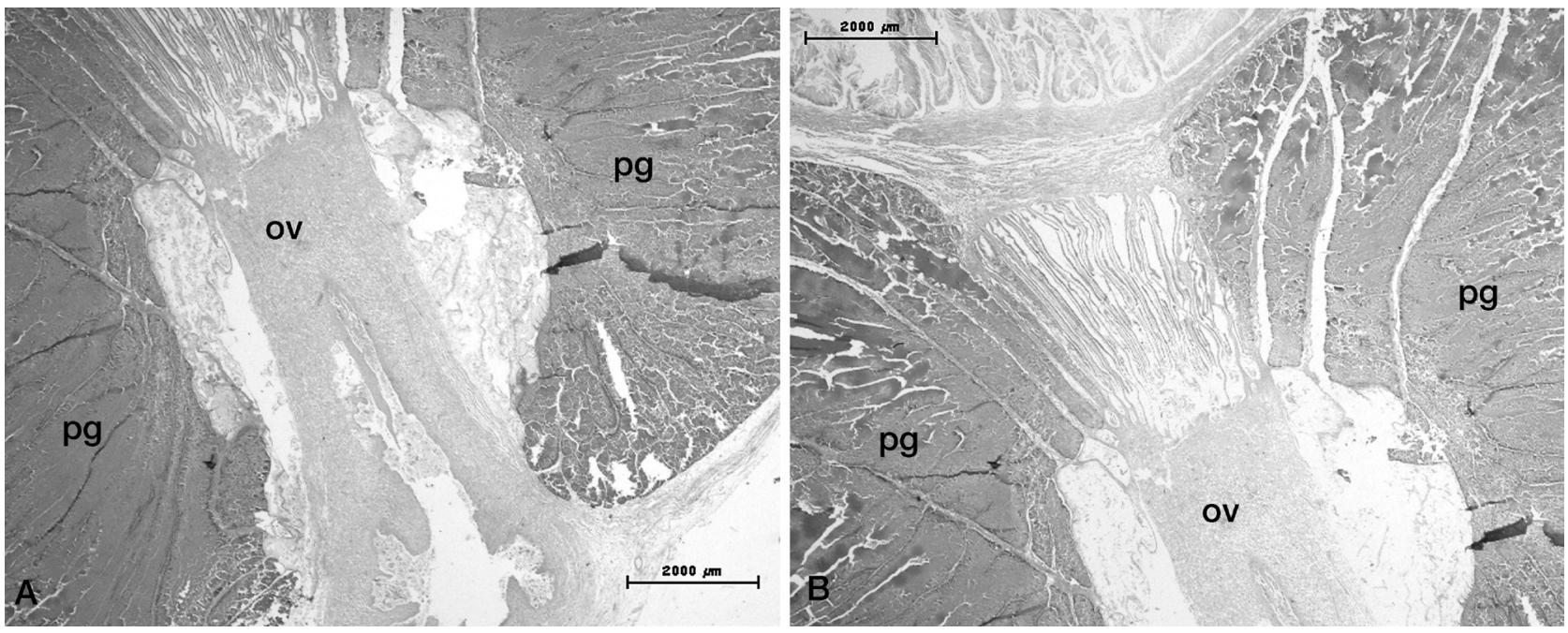

FIG. 10. - Graneledone macrotyla. Longitudinal sections of the oviducal gland (specimen 47); ov, oviduct; pg, peripherical gland.

Eggs large, mature oocytes $20.5-25.5 \mathrm{~mm}$ long (EgLI 18.6-21.1), wide (EgWI 8.7-10.7), and produced in relatively low numbers (263 in specimen MDMG68C2011, Table 3). All five ovaries contained oocytes. Oocytes in four different developmental stages. Immature ovaries (specimens MDMG 94A2011 and 94B2011) contained immature oocytes, which have a smooth surface with some brown spots and different sizes (maximum length 1.0-3.0 mm). Maturat- ing ovaries (specimens MDMG 552011 and 68C2011) contained immature, maturing (3.1-12.8 mm) and fully mature oocytes of different sizes (18.8-25.5 mm). Mature ovaries contained fully mature oocytes, and few (3-4) fertilized eggs $(18.8-20.5 \mathrm{~mm})$. All oocytes at these stages showed a rigid and translucent outer cage with longitudinal striae, narrow micropyle at the distal pole, and long peduncles (Fig. 9C); they were attached to the ovary like clusters of grapes. The fertilized eggs 
were oval in shape (similar to a hen's egg), external capsule hardened, smooth and beige in colour, no micropyle, and very short peduncles (Fig. 9D). Oviducal gland in the mid-portion of each oviduct, formed like a huge concentric peripherical gland around the oviduct and separated by a thin sheet of connective tissue; spermathecae absent. Peripherical gland is formed by groups of concentric cells with basal nuclei and a central lumen; in females close to maturity the cytoplasm is densely packed with reddish grains (Fig. 10). Spermatozoa not seen associated with oviducts. No spermatangia or free sperm within ovaries or attached to egg filaments.

Body sculpture distinctive. Low, thin, peripherical keel around mantle. Dorsal surface of mantle above keel, head and basal part of arms covered by distinct complex papillose warts $3-10 \mathrm{~mm}$ in diameter, consisting of a pale raised area bearing small simple 4-10 tubercles (papillae) generally surrounding one erect, central, sharp tubercle. Ten to twelve wart clusters across dorsal mantle. Warts extend onto web and bases of arms for approximately $15 \%$ of arm length, except on arms 3 and 4 where warts are absent. Row of papillose warts around each eye. No papillose warts on ventral surface of mantle and head. Base colour on dorsal surface of animal ranges from brownish red to purple. Colour is paler on oral surface. Raised pale areas creamy brown.

Distribution. Figure 11 shows the records of $G$. macrotyla described in the present paper, the two records described by Kubodera and Okutani (1994), and the holotype (Voss 1976). Our records extend the known distribution from $45^{\circ} 44^{\prime} \mathrm{S}$ to $45^{\circ} 08.02^{\prime} \mathrm{S}$ and the known depth range from 475 to $2044 \mathrm{~m}$, where the holotype was caught (Voss 1976).

\section{DISCUSSION}

Within the genus Graneledone Joubin 1918, G. macrotyla has an unusual wart structure that makes it very distinct from the other seven species of the genus (Guerra et al. 2000; Allcock et al. 2003). The diagnostic characters of the specimens described here agree with those indicated by Voss (1976) and Kubodera and Okutani (1994). Morphometric measurements and counts are generally within the ranges given by these authors. The discrepancies observed could be due to conservation. This is the first time that stylets, beaks and spermatophores are described, and also the first time that mature females were found. The asymmetry observed in the stylets might be due to body distortion caused by freezing. The radula structure of $G$. macrotyla was variable (homodont and heterodont); the rachidean tooth showed one, two or three cusps depending on its location in the radular ribbon. The use of the radula as a diagnostic character in cephalopods to the generic and specific levels is still controversial, mainly because the radula shows a high individual degree of variation that

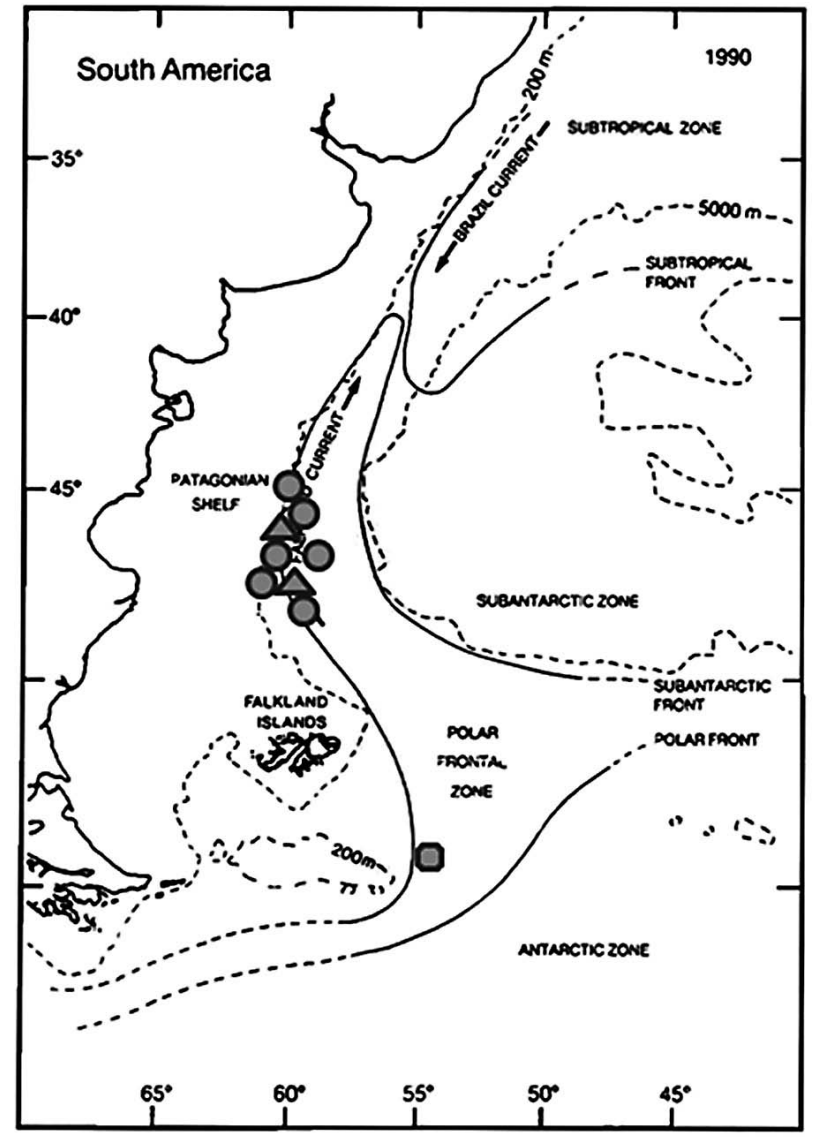

FIG. 11. - Distribution map of Graneledone macrotyla. Square, holotype; triangles, specimens of Kubodera and Okutani (1994); circles, present records.

is evident with both light and scanning electron microscope (e.g. Adam 1941 in different octopod species; Allcock et al. 2003 within the genus Graneledone). Nixon (1998) observed this problem and suggested a number of morphometric studies to ascertain the use of the radula as a diagnostic character; however, they have not yet been carried out. For the above reasons, we decided not to include the shape of the radula as a diagnostic character in G. macrotyla.

The absence of spermathecae in the oviducal glands was observed in four species of Eledone (Perez et al. 1990). This is the first time that this character has been shown in a Graneledone species. Voss and Pearcy (1990) reported that the distal oviduct of Graneledone pacifica contained sperm. However, given that no spermatangia or free sperm were observed within the ovaries of this species, the presence of fertilized eggs inside the ovary suggests that fertilization takes place within the ovary, as observed in Eledone massyae, $E$. gaucha and E. cirrhosa (Perez et al. 1990). This fact also suggests that, as occurs in the two South American eledoninids, G. macrotyla males become mature early and remain sexually active for a greater part of their life than females. It is also possible that females of this species can copulate a considerable number of times before maturation and store sperm until fertilization 
shortly before spawning. It is still unknown where the sperm is attached to the ovaries.

The existence of oocyte cohorts at different oogenic stages suggests a continuous spawning reproductive strategy, in which ovulation is characterized by continuous asynchronous production of ova in the ovary after spawning has commenced. In this monocyclic spawning pattern, adults spawn continuously during their relatively extended life spans, egg-laying occurs in separate batches, and somatic growth takes place during spawning events (Rocha et al. 2001). This strategy is also followed by several cirroctopods and some species of octopods inhabiting deep cold waters (e.g. Bathypolypus arcticus and B. piscatorium) as well as Graneledone pacifica (Bello 2006). A continuous spawning strategy is common in extremely stable environments, and could be an adaptive response to 'safe' environments where predation pressure and competition for resources may be very low. In such environments, spawning can be slow and continuous, with a limited number of eggs per clutch (Rocha et al. 2001).

It is noteworthy that there were numerous oocytes with translucent, longitudinally striated outer cases and long peduncles, but with outer widely opened cases in the distal pole and with a flaccid consistency (Fig. 9D). The high salt content of marine invertebrate cells leads to extensive cellular damage during freezing (Dixon et al. 2002), which induces artificial cleavage of apoptosis-related proteins in human bone narrow (Schmidt-Mende et al. 2000). Atresia has been observed in several species of cephalopods (Melo and Sauer 1998; Boyle and Chevis 1991; Laptikhovsky 2001; Laptikhovsky and Arkhipkin 2001), and massive atresia is more common than we might think, particularly in fish (Rideout and Tomkiewicz 2011). Further analyses will be needed to elucidate whether the appearance of these oocytes is a matter of apoptosis or massive atresia. However, our opinion is that it is most likely an artefact produced by cell damage due to preservation, since the animals were frozen for 12 and 5 months for ATLANTIS 2009 and 2010 respectively

G. macrotyla is a rare, bathybenthic species known only from a very few specimens captured in the west Scotia Basin (Voss 1976; Kubodera and Okutani 1994). The species is currently recorded from depths of 850$2044 \mathrm{~m}$, but four of our specimens (MDMG742011, 1082011, 472011 and 942011) indicate that the species also lives in shallower waters. From a biogeographical point of view our data show that G. macrotyla inhabits the plume of cold subantarctic waters, which is pushed far into the South Atlantic by the Falkland (Malvinas) Current. This concurs with Strugnell et al. (2008) who showed that the deep-sea lineage of these octopods has its evolutionary origins in Antarctica. We suggest that future sampling should be carried out on both sides of this plume, in regions where there is no Antarctic water, to determine whether this cold-water adapted species is present.

\section{ACKNOWLEDGEMENTS}

We wish to express our great gratitude to the members of the research team who participated in the cruises for their contribution in the collection of the samples. The authors also thank the ship's crew for their professionalism and the courtesy extended to the scientific group during the research cruises. We are also grateful to all the people involved in the surveys, namely the scientific and technical personnel who made this work possible. Finally, we want to express our thanks to Mr. Manuel E. García (Garci) for photos and Drs. Louise Allcock and Giambattista Bello for their comments and corrections, which improved the quality of this paper.

\section{REFERENCES}

Adam W. 1941. Notes sur les Céphalopodes, XV. Sur la valeur diagnostique de la radule chez les Céphalopodes Octopodes. Bull. Mus. R. Hist. Nat. Belgique, XVII, 38: 1-19.

Allcock A. L., Collins M. A., Vecchione M. 2003. A redescription of Graneledone verrucosa (Verrill, 1881) (Octopoda: Octopodidae). J. Mollus. Stud. 69: 135-143.

Bello G. 2006. Signs of multiple spawning in Graneledone pacifica (Cephalopoda: Octopodidae). J. Mar. Biol. Mar. Ass. U.K. 86: 1183-1186.

Boyle P.R., Chevis D. 1991. Changes in follicle cell epithelium nuclei at the onset of vitellogenesis in the octopus Eledone cirrhosa. Bull. Mar. Sci., 49: 372-378.

Dixon D., Priski R.A.M., Dixon L.R.J., Jha A.N. 2002. Marine invertebrate eco-genotoxicology: a methodological overview. Mutagenesis, 17: 495-507.

Furuya H., Hochberg F.G. 2002. New species of Dicyemennea (Phylum: Dicyemida) in deep-water Graneledone (Mollusca: Cephalopoda: Octopoda) from the Antarctic. J. Parasitol. 88: 330-336.

Gabe N. 1968. Techniques histologiques. Masson et Cie, Paris.

Guerra A., González A.F., Cherel Y., Rocha F. 2000. Graneledone gonzalezi sp. nov. (Mollusca: Cephalopoda): a new octopod from the Kerguelen Islands. Antarc. Sci. 12: 33-40.

Guerra A., Portela J.M., del Río J.L. 2011. Cephalopods caught in the outer Patagonian shelf and its upper and medium slope in relation with the main oceanographic features. Fish. Res. 109: 179-186.

Kubodera T., Okutani T. 1994. Eledonine octopods from the Southern Ocean: systematics and distribution. Antarc. Sci. 6: 205-214.

Laptikhovsky V.V. 2001. Fecundity, egg masses and hatchlings of Benthoctopus spp (Octopodidae) in Falkland waters. J. Mar. Biol., Ass., U.K. 81: 267-270.

Laptikhovsky V.V., Arkhipkin, A.I. 2001. Oogenesis and gonad development in the cold water loliginid squid Loligo gahi $(\mathrm{Ce}-$ phalopoda: Myopsida) on the Falkland shelf. J. Mollus. Stud. 67: 475-482

Melo Y.C., Sauer W.H.H. 1998. Ovarian atresia in cephalopods. S. Afric. J. Mar. Sci. 20: 143-151.

Nixon M. 1995. A nomenclature for the radula of the Cephalopoda (Mollusca) - living and fossil. J. Zool. London 236: 73-81.

Nixon M. 1998. The radulae in Cephalopoda. Smith. Contr. Zool. $n^{\circ}$ 586: 39-54.

Perez J.A.A., Haimovici M., Cousin J.C.B. 1990. Sperm storage mechanism and fertilization in females of two South American eledonids (Cephalopoda: Octopoda). Malacologia 32: 147-154.

Rideout, R.M., Tomkiewicz J. 2011. Skipped spawning in fishes: more common that you might think. Mar. Coast. Fish. 3: 176-189.

Rocha F., Guerra A., González A. F. 2001. A review of the reproductive strategies in cephalopods. Biol. Rev. 76: 291-304.

Roper C.F.E., Voss G.L. I983. Guidelines for taxonomic descriptions of cephalopods species. Mem. Nat. Mus. Victoria 44: 49-63.

Schmidt-Mende J., Hellström-Lindberg E., Joseph B., Zhivotovsky B. 2000. Freezing induces artificial cleavage of apoptosis- 
related proteins in human bone marrow cells. J. Immunol. Meth. 245: 91-94

Strugnell J.M., Rogers A.D., Prodöhl P.A., Collins M.A., Allcock A.L. 2008. The thermohaline expressway: the Southern Ocean as a centre of origin for deep-sea octopuses. Cladistics 24: 853-860.

Voss G.L. 1976. Two new species of octopods of the genus Graneledone (Mollusca: Cephalopoda) from the Southern Ocean. Proc.
Biol. Soc. Washington 88: 447-458.

Voss G.L., Pearcy W.C. I990. Two new species of deep water octopods (Mollusca; Cephalopoda) of the northeastern Pacific. Proc. California Acad. Sci. 47: 47-94.

Scient. ed.: P. Sartor.

Received July 29, 2011. Accepted November 28, 2011.

Published online May 7, 2012. 